\title{
A silicon array for cosmic-ray particle identification in space
}

\author{
S. Bonechi*, M.G. Bagliesi, G. Bigongiari, P. Brogi, P. Maestro, P.S. Marrocchesi, \\ F. Pacciani, A. Sulaj \\ Dept. Physical Sciences, Earth and Environment, University of Siena and INFN Pisa \\ E-mail: sbonechiepi.infn.it
}

A. Basti, T. Lomtazde, F. Morsani

INFN Pisa

\begin{abstract}
A new generation of space experiments with very large geometric factors (of the order of 3 - 4 $\mathrm{m}^{2} \mathrm{sr}$ ) are being designed to perform precision studies of the elemental composition of Very High Energy (VHE) cosmic nuclei and of their spectral features. In the current concept of the Gamma400 experiment, the charge identification of the incoming particle is performed by a two-layered array of pixelated silicon sensors. Given the orbital parameters of the mission, the isotropic distribution of the incoming cosmic rays can be sampled on the 4 lateral sides of the instrument. The conceptual design of the array, covering a seamless sensitive area of the order of $1 \mathrm{~m}^{2}$, will be presented together with results obtained with reduced-scale prototypes at relativistic ion beams.
\end{abstract}

11th International Conference on Large Scale Applications and Radiation Hardness of Semiconductor Detectors

3-5 July 2013

Auditorium Cassa di Risparmio di Firenze, via Folco Portinari 5, Florence, Italy

\footnotetext{
* Speaker.
} 


\section{Introduction}

An accurate measurement of electric charge of fully stripped ions allows the identification of the chemical elements in the cosmic-ray $(\mathrm{CR})$ spectrum. Experiments that measure the chemical composition and the spectra of CR have been performed in space with satellites or on board of the ISS and at ground level with large area telecopes. In the latter case, the primary nuclei are not identified directly, but their nature is inferred studying their interactions with the atmosphere, resulting in the introduction of sizable systematic errors. On the contrary, experiments in space are free from residual interactions and a direct detection of VHE CR in the knee region (around $10^{15} \mathrm{eV}$ ) is possible, in case of a detector with large geometrical factor and a long exposure. In this scenario the GAMMA-400 instrument [四] will be installed on the Russian spacecraft "Navigator", the planned long duration of the mission (about 10 years) and the orbital parameters (perigee $100,000 \mathrm{~km}$, apogee $200.000 \mathrm{~km}$ ) provide an excellent environment to study, not only gamma-rays and electrons, but also the elemental composition of cosmic rays, in particular the measurements of secondary-to-primary abundances in the cosmic-ray flux (e.g. B/C, sub-Fe/Fe). These observables provide important information on propagation models of VHE CRs. In fact abundance ratios are known to decrease, following a power-law in energy $E^{-\delta}$, where $\delta$ is the diffusion coefficient of CRs propagation in the Galaxy. An accurate measurement of the parameter $\delta$ is crucial to derive the energy spectrum at the source by correcting the observed spectral shape for the energy dependence of the propagation term.

\section{The Silicon Array detector}

In an experiment for the direct detection of cosmic rays at the $\mathrm{TeV}$ scale, the charge detector is affected by the back-scattered shower particles emerging from the calorimeter, which may induce fake charge identification by releasing additional amounts of energy besides the primary particle ionization signal. The choice of a pixel segmentation allows to reduce the probability that the back-scattered particles cross the same pixel as the primary CR originating the shower, and to identify and eliminate the hits not associated to the incident particle track. Arrays of silicon detectors have been successfully operated as charge identifiers both in the ATIC and in the CREAM balloon missions, where they achieved an adequate discrimination capability to tag individual elements, with excellent charge resolutions. Another important feature is the requirement to have two independent charge measurements of the same particle by means of a two-layered Silicon Array (SIA). As demonstrated in the case of the second flight of CREAM ([[3]), the request of a consistent charge measurement from both layers, provides a great benefit in terms of background rejection to increase the purity of the data sample when a single element is selected.

Of particular interest is the measurement of the flux ratio of secondary to primary elements, in particular boron to carbon and iron to sub-iron, that can provide information on interstellar transport parameters. Unfortunately, in case of atmospheric experiments the systematic uncertainty due to the irreducible background of the atmosphere does not allow the discrimination between different models. A two-layered array of pixelated silicon sensors designed for Gamma-400 experiments will perform good measurements on CR nuclei spectra.

The SIA detector proposed for GAMMA-400 consists of a double-layer of silicon matrix wafers 
covering a sensitive area of about $1 \mathrm{~m}^{2}$ with no dead regions. The idea is to assemble different ladders, where in each ladder we have 10 pixellated detector modules with two matrix sensors assembled to achieve a full overlap (see the left panel of figure $\mathbb{W}$ ). Each matrix sensor is segmented on one-side with 64 pixels of dimension $11.25 \times 11.25 \mathrm{~mm}^{2}$ with inter-pixel distance of $0.1 \mathrm{~mm}$. The sensors are produced from 6 inches wafers of $500 \mu \mathrm{m}$ thickness with high resistivity ( $>10$ $\Omega \cdot \mathrm{cm}$ ). The full depletion voltage is below $30 \mathrm{~V}$. The dark current per pixel is typically lower than $1 \mathrm{nA}$ and the capacitance per pixel is $25 \mathrm{pF}$ at full depletion[四].

The Detector Module is composed by a pairs of sensors overlapped and read-out by a front end VA

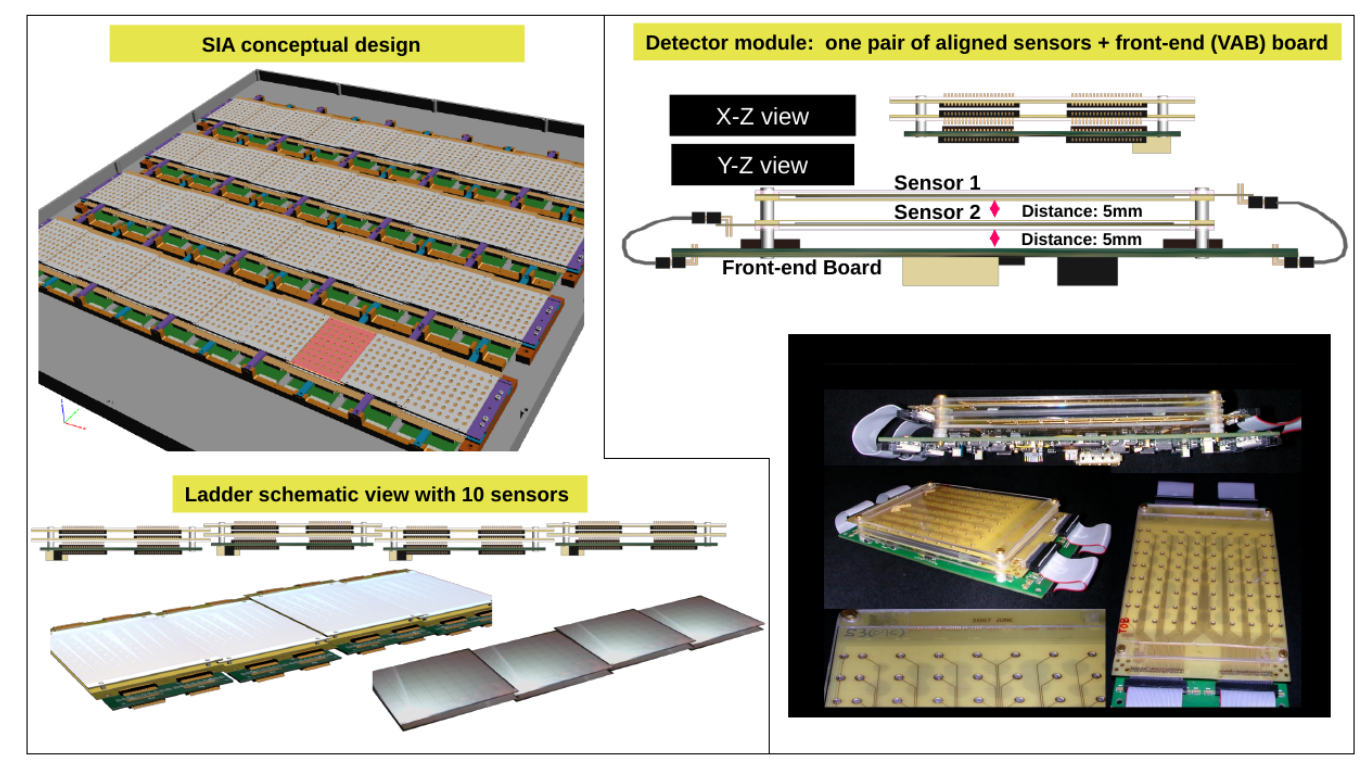

Figure 1: Conceptual design of Silicon Array (SIA) proposed for GAMMA-400.

board. On the right panel of figure $\square$ an early prototype of the Detector Mudule for ground tests is shown. VA Board (VAB) is equipped with 4 ASIC of the VA family, each reading out 32 channels. The VAB is equipped with two FPGA for the readout of 4 VA chips with 16 bits digitization, using 4 independent ADC units[[]]. The global readout of the ladders is done by a ReadOut Controller board (ROC) interfaced to the main Data AcQuisition (DAQ) system and trigger. Redundance will be implemented in the Space VA Board (SVAB).

\section{Beam test of the prototype}

Measurements with prototypes of the matrix sensors were performed in a dedicated beam test that took place at CERN in January and February 2013. Relativistic ions were extracted as secondary products from the interactions of a primary fully stripped $\mathrm{Pb}$ beam at the SPS (with a mean intensity of $\sim 8 \cdot 10^{7}$ per spill) impinging on an internal $300 \mathrm{~mm}$ thick Be target. Fully ionized nuclear fragments with $A / Z=2$, ranging from deuterium to heavy nuclei with atomic number $Z>26$, were steered along the $\mathrm{H} 8$ beam line of the SPS, where the apparatus was configured as summarized in the following. More than 15 million triggers were collected in two sets of runs with beam energies of 13 and $30 \mathrm{GeV} / \mathrm{amu}$, respectively. 


\subsection{Beam test setup}

A beam trigger was provided by the signal coincidence between two plastic scintillators, whose overlap area is approximately a square with $5 \mathrm{~cm}$ side. Besides this physical trigger, a random trigger was provided by mean of a pulse generator: a square wave signal with $100 \mathrm{~Hz}$ frequency was enabled during time intervals between two consecutive beam spills. The main goal of the random trigger is to collect pedestal data, i.e. the average ADC value corresponding to input signals which are compatible with the electronic noise. The pedestal was measured and subtracted from the collected ADC counts in order to obtain a value which is proportional to the corresponding channel pulse height. Since pedestals can fluctuate because of several causes (e.g. electromagnetic interference with other instruments and variations in the leakage current due to thermal effects), a careful pedestal monitoring is needed in order to perform the offline data correction.

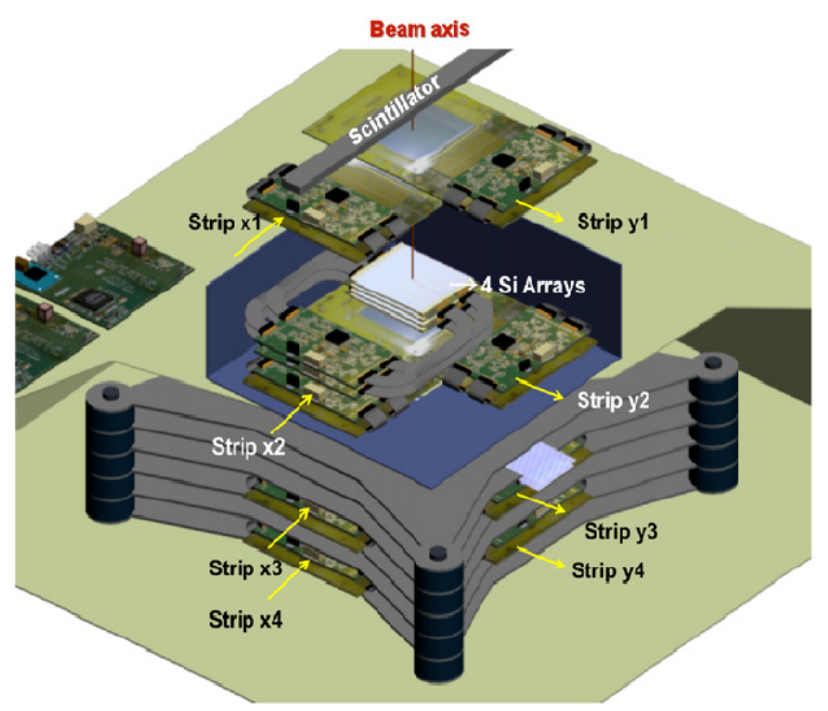

Figure 2: Beam tracker configuration.

The Beam Tracker (BT) is equipped with 2 detector module of SIA to ensure a good reconstruction of the charge. In order to precisely measure the trajectory of the incoming particle, the tracker contains also four pairs of X-Y layers made of silicon strip sensors, as shown in Figure ․ Each layer is $9.4 \mathrm{~cm} \times 9.4 \mathrm{~cm}$ and contains 128 ribbons; each ribbon is $732 \mu \mathrm{m}$ wide and is obtained by ganging four strips (each one with a $183 \mu \mathrm{m}$ pitch). In this configuration, the reconstructed position resolution is better than $200 \mu \mathrm{m}$. The trajectory was reconstructed by means of four independent position measurements for each view.

\subsection{Data Analysis}

Data analysis involves the track reconstruction of the event using the silicon strip detectors to study the response of the double layer matrix to the ion beam. An example of a reconstructed track is shown in figure [3. A clustering algorithm was performed for each strip layer to identify the hits associated to the track. Then for each view (x,y) a combinatorial algorithm produces different tracks associated with the selected hits. At the end, the physical track can be identified by means 


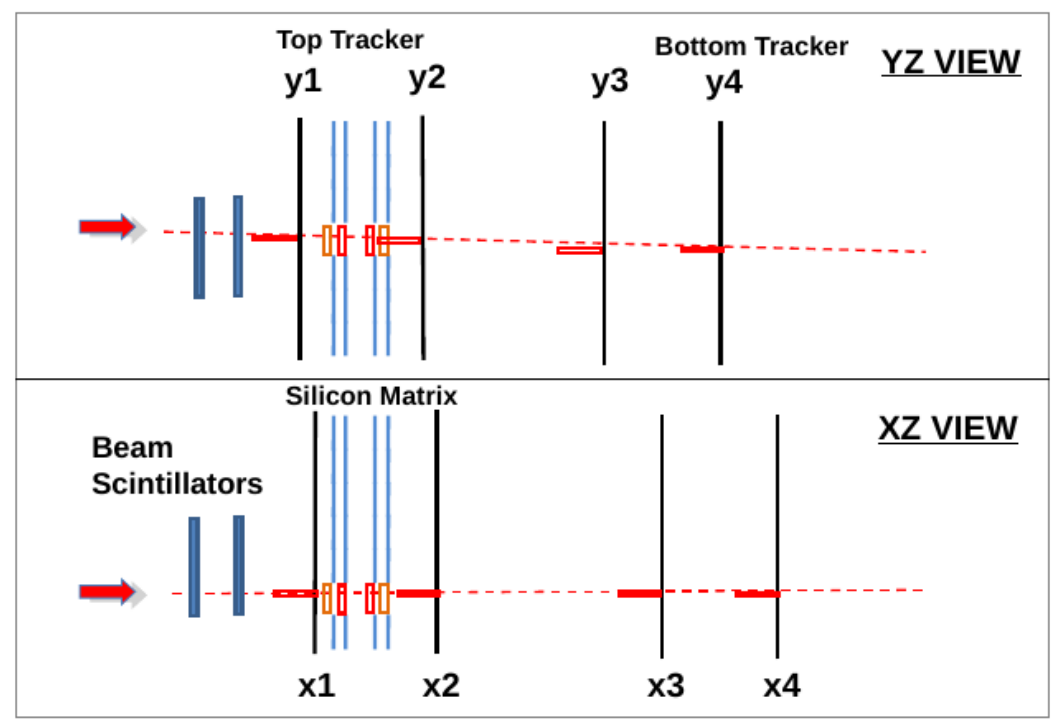

Figure 3: An event reconstructed in beam tracker.

of a $\chi^{2}$ criterion. Using the track parameters it was possible to perform an accurate calibration of each matrix pixel selecting the one crossed by the beam particle. Knowing the value of the digital counts (ADC) for a MIP particle of charge $\mathrm{Z}=1$ [ [6] the calibration was performed estimating the response of each layer to different nuclei by means of Gaussian fits. Once we have fitted all the peaks, we can plot the mean value of each peak versus $Z$ and then perform a parametrization for each matrix layer with the response function $S(A D C)=a+b Z+c Z^{2}+d Z^{3}+e Z^{4}$. The third and fourth order terms, as we expected, are not so significant. The guideline was to transform the ADC response of each detector into a charge variable $Z$ by inverting the response function $S(A D C)$.

A physical process that can worsen the resolution of silicon matrix is the interaction of a nucleus inside the BT. A nucleus that has been tagged as $Z$ in the first double layer of Strips could have interacted inside BT and tagged by the last two strip layers as a nucleus of charge $Z-1, Z-2, \ldots$ (see figure $\$$ (a)). In order to obtain a data sample free from interacting events a procedure using a selection criteria was elaborated. The basic idea is to perform a cut in the two-dimensional correlation plot between the mean $Z$ signal of the last two strip layers $S_{78}^{S T R}$ and the mean $Z$ signal of the first two strips layers $S_{12}^{S T R}$. The variable on which the cut was performed is the asymmetry $A$ between $S_{12}^{S T R}$ and $S_{78}^{S T R}$, defined as:

$$
A=\frac{S_{78}^{S T R}-S_{12}^{S T R}}{S_{78}^{S T R}+S_{12}^{S T R}}
$$

whose distribution is shown in figure田(b). The critical interval chosen for this cut is $A \in[-0.07,0.07]$. With this cut the presumed interacting events were, in the large part, removed from our data sample.

Using the reconstructed track for each event the crossed pixel was selected. Similarly, this procedure will be applied to GAMMA-400 data analysis. The selection of the crossed pixel reduces the probability of a wrong charge identification due to the backsplash deposits coming from the calorimeter. This effect, that is practically negligible at the beam test energy, will be studied with dedicated MC simulations at a flight energy of $1 \mathrm{TeV} / \mathrm{amu}$. 

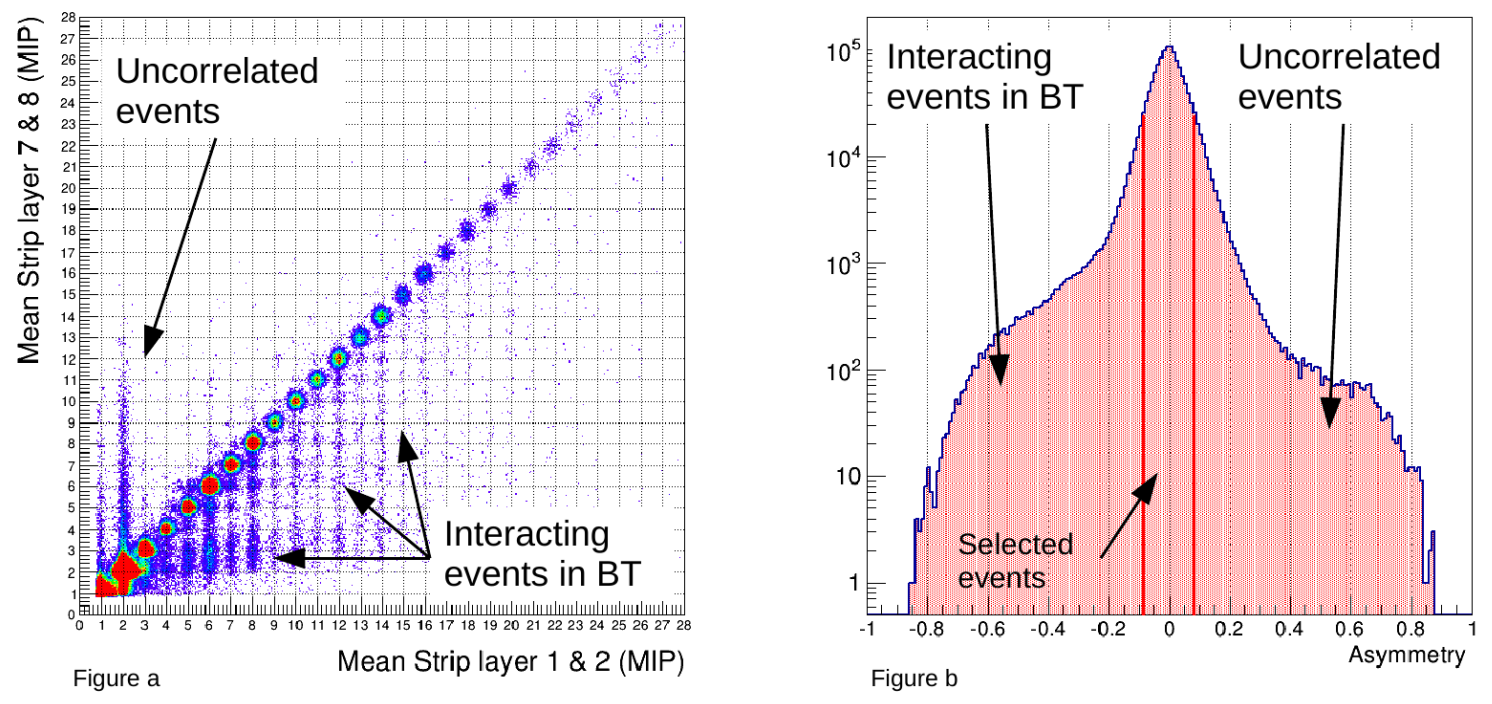

Figure 4: Figure a: correlation plot between the average of the signals from the last two layers vs the average of the first two. Figure b: asymmetry plot to remove interacting and uncorrelated events.

The double silicon matrix layer has the capability to identify the charge $Z$ from proton to iron and beyond (figure $\square$ (a)) with a good charge resolution. Combining the information of the two layers, we get a charge resolution of $\approx 0.16 e$ fulfilling the requirement for a $5 \sigma$ separation of boron from carbon (figure 5 (b)). For heavier nuclei the charge resolution does not worsen much more than $0.25 e[\square]$. This allows to separate consecutive elements in the periodic table with a small probability of misidentification, as it is essential for the measurements of secondary-to-primary abundances in the cosmic-ray flux (e.g. B/C, sub-Fe/Fe).
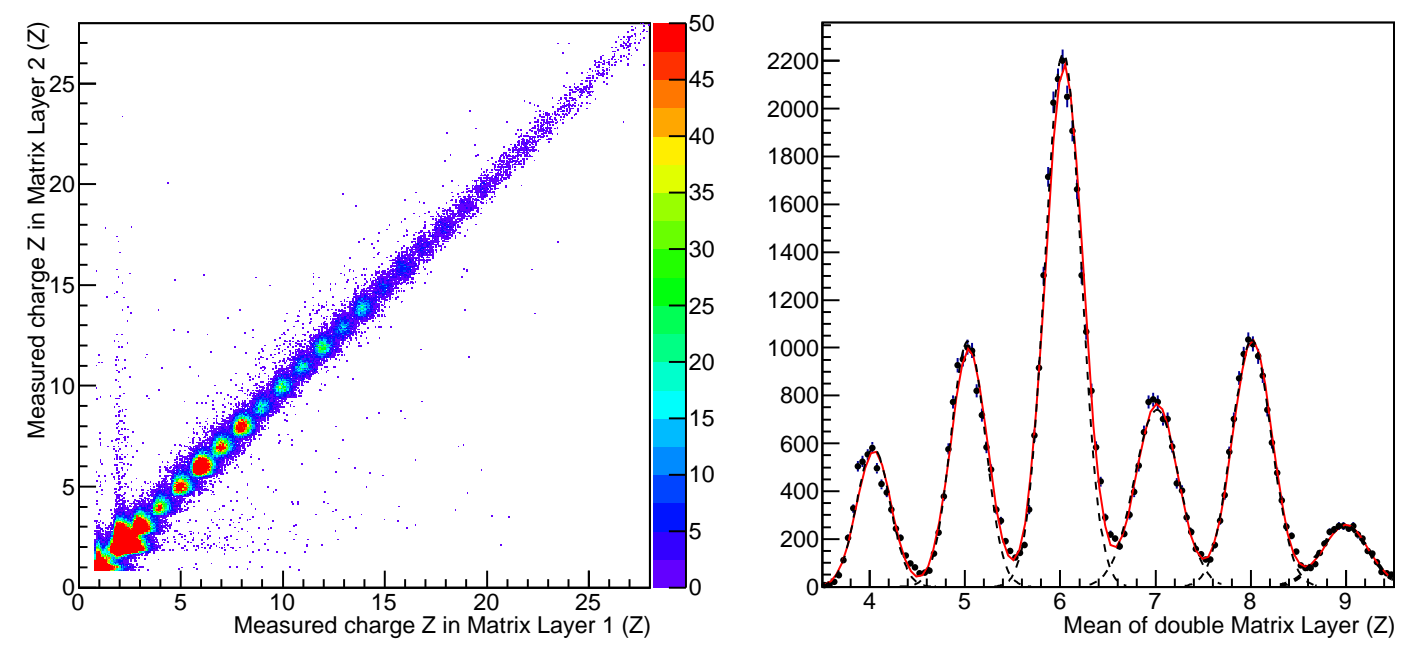

Figure 5: Figure a: correlation plot between the signal in matrix 2 vs matrix 1. Figure b: Mean signal of the double matrix layer for Be-B-C-N-O-F group. 


\section{Conclusions}

The charge detector prototype and the associated electronics were capable to provide an excellent charge identification of relativistic nuclei. The dynamic range of the front-end electronics was properly designed to allow a linear response of the system up to the largest charges under test. The correlation of the signals from two silicon strip layers of the BT, placed at different depths in the telescope, proved to be a powerful tool to identify nuclei interacting in the detector material and allowed to reject them in the data analysis. Moreover, by combining the response of two separate layers of a $\mathrm{Si}$ array, an excellent charge resolution in the range of $0.16 e-0.25 e$ was achieved from Boron to Iron.

\section{References}

[1] A.M. Galper et al., http://arxiv.org/abs/1306.6175

[2] E. Mocchiutti et al., Nuclear Physics B (Proc. Suppl.) 239-240 (2013) 204-209

[3] Marrocchesi P.S et al.. et al., Advances in Space Research, Volume 41, Issue 12, 2008, 2002-2009

[4] M.Y. Kim et al., RD09_035 http://pos.sissa.it/archive/conferences/098/035/RD09_035.pdf

[5] M.G. Bagliesi et al., Nuclear Physics B - Proceedings Supplements 01/2007

[6] Marrocchesi P.S. et al., Proceedings of the 30th International Cosmic Ray Conference

[7] P. Maestro et al., Nuclear Instruments and Methods in Physics Research A 679 (2012) 7-13 